\title{
An improved NAS-RIF Algorithm based on Wavelet Packet
}

\author{
Wael M. Khedr \\ Zagazig University of Science, \\ Zagazig, Egypt
}

\author{
Mohamed S. Sawah \\ Computer Science Instructor at \\ Science Valley Academy, \\ Cairo, Egypt
}

\begin{abstract}
Image restoration is widely applied in many areas. When operating on images with different scales for the representation of pixel intensity levels or low SNR, the traditional restoration algorithm lacks validity and induces noise amplification, ringing artifacts and poor convergent ability. In this paper, an improved NAS-RIF algorithm is proposed to overcome the shortcomings of the traditional algorithm. The improved algorithm proposes a solution for blurred with noise image by constrained maximization of some of the detail wavelet packet energies. This algorithm gives enhancement with the sharpness of the deconvolved images. In determining the support region, a presegmentation is used to form it close to the object in the image, Moreover, as compared with the traditional algorithm. Simulations show that the improved algorithm behaves a better convergence, noise resistance and provides a better estimate of the original image.
\end{abstract}

\section{Keywords}

Blurred image, NAS-RIF algorithm, Image Restoration, Point Spread Function (PSF), Conjugate Gradient (CG), Peak Signal to Noise Ratio (PSNR) and wavelet packet function.

\section{INTRODUCTION}

In many imaging applications, the degradation of the true image can be modeled as the model consists of the observed image $\boldsymbol{g}(\boldsymbol{x}, \boldsymbol{y})$, True image $\boldsymbol{f}(\boldsymbol{x}, \boldsymbol{y})$, And noise $\boldsymbol{g}(\boldsymbol{x}, \boldsymbol{y})$ that is coupled linearly; therefore, the problem of recovering $\boldsymbol{f}(\boldsymbol{x}, \boldsymbol{y})$ from $\boldsymbol{g}(\boldsymbol{x}, \boldsymbol{y})$ is referred to as the liner image restoration problem. The existing linear image restoration algorithms assume that the PSF is known a priori and attempt to invert it and reduce noise by using varying amounts of information about the PSF, true image, and noise statistics [1].

In many situations, however, the PSF is unknown, and little can be assumed about the original image. Therefore, the majority of existing linear image restoration techniques are not applicable for solving this type of problem. The process of simultaneously estimating the PSF (or its inverse) and restoring an unknown image using partial or noinformation about the imaging system is known as blind image restoration [4].

For the linear degradation model, where the noise term $n(x, y)$ is neglected, it is specifically referred to as blind deconvolution [5] there exist several motivating factors for the use of blind deconvolution in image processing applications. In many situations, it is difficult to accurately measure the degradation using calibration or on-line identification techniques; in addition, it is Costly, dangerous, or physically impossible to obtain a priori information about the scene to be imaged. For example, in remote sensing and space imaging, fluctuations in the PSF are difficult to characterize as a random process, and there is difficulty in statistically modeling the original image [2].

In practice, some a priori information is required to restore the image successfully. The partial information available is specific to each imaging application; therefore, many diverse techniques for blind deconvolution of images have been proposed. The challenge is to design a method that exhibits the most appropriate compromise among computational complexity, reliability, and robustness to noise. NAS-RIF algorithm [4] is applied on blurred image without noise through determine the support non-negative region, a presegmentation to close to the object in the image. We improve this algorithm to restore true image from the blurred noisy image. We provide a wavelet packet detail over the traditional NAS-RIF algorithm to enhance with the sharpness of the deconvolved image and update the direction parameter of conjugate gradient method. The only information required for restoration is the non-negativity of the true image and support size of the original object. The restoration procedure involves recursive filtering of the blurred image to minimize a convex cost function.

The traditional NAS-RIF algorithm is proposed in section 2. Our contributions of this paper are developed by NAS-RIF algorithm for the restoration of linearly degraded images in section 3. The proposed technique is applied to applications in which an object of finite extent is imaged against a uniform black or white background. The edges of the object are assumed to be completely or almost completely included within the observed frame. This often occurs in some types of astronomical imaging, medical imaging, among others. The advantage of the proposed technique over existing methods is that convergence to the feasible set of solutions is guaranteed with high performance PSNR.

\section{NAS-RIF ALGORITHM}

The proposed method is referred to as the non-negativity and support constraints recursive inverse filtering (NAS-RIF) algorithm. The blurred image $\boldsymbol{g}(\boldsymbol{x}, \boldsymbol{y})$ is input to a 2-D variable coefficient FIR filter $\boldsymbol{u}(\boldsymbol{x}, \boldsymbol{y})$ whose output represents an estimate of the true image dented $f^{\wedge}(x, y)$.This estimate passes through a non-linear constraint process that uses a nonexpansive mapping to project the estimated image into the space representing the known characteristics of the true image. The difference between the projected image $f^{\wedge}{ }_{N L}(x, y)$ and $f^{\wedge}(x, y)$ is used as an error signal to update the coefficients of filter $\boldsymbol{u}(\boldsymbol{x}, \boldsymbol{y})$ Fig. 2 gives an overview of the method. For the NAS-RIF algorithm, the image is assumed to be non-negative with known support; therefore, $f^{\wedge}{ }_{N L}(x, y)$ represents the projection of the estimated image onto the set of images that are non-negative with given finite support. This requires replacing the negative pixel values within the region of support with zero and pixel values outside the region of support with the background gray-level value $L_{B}$. The problem is formulated by (1), 


$$
g(x, y)=f(x, y) * h(x, y)+n(x, y)
$$

Where: $\boldsymbol{g}(\boldsymbol{x}, \boldsymbol{y})$ Is blurred image, $\boldsymbol{f}(\boldsymbol{x}, \boldsymbol{y})$ True image, $\boldsymbol{h}(\boldsymbol{x}, \boldsymbol{y})$ represents Point spread function (PSF), and $\boldsymbol{n}(\boldsymbol{x}, \boldsymbol{y})$ Is an additive noise. The ' $*$ ' is discrete (2-D) linear convolution operator.

The objective of blind image deconvolution is to construct a reliable estimate of the imaged scene from a blurred version.

In this paper, NAS-RIF constraints about the imaging process, the true image, and the PSF.

1) The degradation is described by the linear model of (1).

2) Imaging is performed such that the object is entirely encompassed by the observed frame.

3 ) The background of the image is uniformly gray, black, or white.

4) The true image is nonnegative, and its support is known a priori; the support is defined to be

The smallest rectangle encompassing the object of interest (Fig. 2 illustrates the region of

Support).

5) The true image and PSF are irreducible; the term irreducible refers to a signal that cannot be

Expressed as the convolution of two or more component images of finite support on the

Understanding that the delta function is not a component image.

6) The inverse of the PSF exists, and both the PSF and its inverse are absolutely assumable that

Is,

$$
\sum_{x=-\infty}^{\infty} \sum_{y=-\infty}^{\infty}|h(x, y)|<\infty
$$

And

$$
\sum_{x=-\infty}^{\infty} \sum_{y=-\infty}^{\infty}\left|h^{-1}(x, y)\right|<\infty
$$

7) In the situation where the background of the image is black, the sum of all PSF pixels is

Assumed to be positive, which occurs in almost all image processing applications.

The cost function used in the restoration procedure is defined as

$J=\sum_{\forall(x, y)}\left[f^{\wedge}(x, y)-f^{\wedge}(x, y)\right]^{2}$

Where

$$
\begin{aligned}
& \hat{f}_{N L}(x, y) \\
& = \begin{cases}\hat{f}(x, y) & \text { if } \hat{f}(x, y) \geq 0 \text { and }(x, y) \in D_{\text {sup }} \\
0 & \text { if } \hat{f}(x, y)<0 \text { and }(x, y) \in D_{\text {sup }} \\
L_{B} & \text { if } \hat{f}(x, y) \in 0 \text { and }(x, y) \in \bar{D}_{\text {sup }}\end{cases}
\end{aligned}
$$

Using

$(4)$,

$$
\text { (3) }
$$

reduces

$$
\begin{aligned}
J=\sum_{(x, y) \in D_{\text {sup }}} \hat{f}^{2}(x, y) & {\left[\frac{1-\operatorname{sgn}(\hat{f}(x, y))}{2}\right] } \\
+ & \sum_{(x, y) \in \bar{D}_{\text {sup }}}\left[\hat{f}(x, y)-L_{B}\right]^{2}(4)
\end{aligned}
$$

Where the definition of sign (.)

$$
\operatorname{sgn}(f)= \begin{cases}1 & \text { if } f \geq 0 \\ -1 & \text { if } f<0\end{cases}
$$

$D_{\text {sup }}$ is the set of all pixels inside the region of support, and $\bar{D}_{\text {sup }}$ Is the set of all pixels outside the region of support. As we can see, for situations in which the background is black $L_{B}$, The parameter sets $u(x, y)=0$ for all $(x, y)$ globally minimizes $J$. This results in a restored image $\hat{f}(x, y)=0$ for all $(x, y)$, Which is the all black solution. To avoid this trivial solution, we make use of above constraint 7 , which states that the sum of all the PSF coefficients is positive, i.e.,

$$
\sum_{\forall(x, y)} h(x, y)=S_{h}>0
$$

Using the fact that the 2-D discrete-time Fourier transform of $h(x, y)$ isgiven by

$$
H(0)=\sum_{\forall(x, y)} h(x, y)
$$

We can deduce that

$$
H(0)=S_{h}>0
$$

Taking the reciprocal of both sides and $h_{i n v}(x, y)$ and $H_{i n v}(w)$ be the spatial and Fourier transform functions of the ideal inverse of $h(x, y)$,respectively, we see that

$$
H_{i n v}(0)=\frac{1}{H(0)}=\frac{1}{S_{h}}
$$

Or, effectively

$$
\sum_{\forall(x, y)} h_{i n v}(x, y)=\frac{1}{S_{h}}>0
$$

Thus, we can deduce that the sum of the pixels of the inverse PSF is also positive. We can use this fact to constrain the parameters $\{u(x, y)\}$ from the trivial all-zero solution. Since our goal is to obtain a positive scaled version of the ideal image $f(x, y)$, we can constrain the sum of all the filter coefficients $u(x, y)$ to be any positive constant to meet this objective. In this paper, we choose $S_{h}=1$ so that we have the following constraint on our FIR filter coefficients:

$$
\sum_{\forall(x, y)} u(x, y)=1
$$

The NAS-RIF algorithm did not yield results as expected. NAS-RIF algorithm makes use of constraints only in spatial domain, not in spatial domain. That may be the reason for the unsatisfactory performance. Minimizing the cost function may result in one of several solutions, most of which are physically meaningless to the problem .The major advantage of the algorithm is that it entails the minimization of a convex cost function.

\section{IMPROVED NAS-RIF ALGORITHM.}

In the implementation of the NAS-RIF algorithm, we use the iterative conjugate gradient minimization routine. One option for constraining the parameters to fulfill (11) is to normalize $u(x, y)$ atevery iteration. The cost function consists of three components. The first penalizes the negative pixels of the image estimate inside the region of support, and the second penalizes the pixels of the image estimate outside the region of support that are not equal to the background color. The third component is used to constrain the FIR filter coefficients $u(x, y)$ away from the trivial all-zero global minimum. The conjugate gradient minimization routine is used for the 
minimization of $\mathrm{J}$ because its speed of convergence is, in general, much faster than other descent methods .The deblurred image quality can be improved through boosting the energies of the wavelet packets. This goal can be achieved by processing these sub-bands by a $2 \mathrm{D}$ mask $H_{0}$ that optimizes their energies while minimizing the residual energy of $J_{e r}$

$J_{e r}(x, y)=f_{k}(x, y)$

$$
-\sum_{\theta=0}^{N_{1}-1} \sum_{l=0}^{N_{1}-1} \hat{h}(x, y) \hat{f}(\mathrm{x}-\theta, y
$$

Improved NAS-RIF Algorithm

- $\quad$ Set initial conditions $(\mathrm{k}=0)$ :

FIR filter:

$u^{T}{ }_{k}=\left[u_{k}(1,1), \ldots ., \frac{u_{k}\left(N_{x u}+1\right)}{2}, \frac{N_{y u}+1}{2}, \ldots, u_{k}\left(N_{x u}, N_{y u}\right)\right]=$ $[0, \ldots, 1, \ldots, 1]$

Tolerance $\partial>0$ is set.

- At iteration $(k): k=0,1,2, \ldots$

1) If $J\left(u_{k}\right) \leq \partial$, stop.

2) Calculate the gradient vector of $J$

a) $f_{k}(x, y)=u_{k}(x, y) * g(x, y)$

b) $\left[\nabla J\left(u_{k}\right)\right]_{j+(i-1) N_{x u}, 1}=\frac{\partial J\left(\underline{u}_{k}\right)}{\partial u(i, j)}=$

$2 \sum_{(x, y) \in D_{\text {sup }}} \hat{f}^{2}(x, y)\left[\frac{1-\operatorname{sgn}(f(x, y))}{2}\right] g(x-$

$i+1, y-j+1)+2 \sum_{(x, y) \in \bar{D}_{\text {sup }}}[\hat{f}(x, y)-$

$\left.L_{B}\right] g(x-i+1, y-j+1)+$

$2 \gamma\left[\sum_{\forall(x, y)} u(x, y)-1\right]$

3) if $k=0, d_{k}=-\nabla J\left(u_{k}\right)$.

Otherwise,

a) $\quad \beta_{k}^{N}=\left(y_{k}-2 d_{k} \frac{\left\|y_{k}\right\|^{2}}{d_{k}^{T} y_{k}}\right)^{T} \frac{g_{k+1}}{d_{k}^{T} y_{k}}(2005)$ proposed Hager and Zhang.

b) $d_{k}=-\nabla J\left(u_{k}\right)+\beta_{k} d_{k}$

4) Perform a line minimization :

$$
J\left(u_{k}+t_{k} d_{k}\right) \leq J\left(u_{k}+t d_{k}\right) \text { for } t \in R
$$

5) $u_{k}+1=\left(u_{k}+t_{k} d_{k}\right)$

6) Then, reconstruct the estimated image $f_{k}(x, y)$ by the convolution with the wavelet packet function $\mathrm{H}$ using the synthesis band to get $J_{e r}(x, y)$. Evaluate the residual error: $J_{e r}(x, y)$

$$
\begin{aligned}
& ={f^{\wedge}}_{k}(x, y) \\
& -\sum_{\theta=0}^{N_{1}-1} \sum_{l=0}^{N_{1}-1} \hat{h}(x, y) \hat{f}(\mathrm{x} \\
& -\theta, y-l)
\end{aligned}
$$

7) Then, we use the built in function deconvblindin matlab to obtain the enhanced image.

\section{APPLICATIONS}

Simulations were carried out by using MATLAB7.8.0.340different three images as (Text, Flower, and Lena) with blurred, plus noise and different types of degradations were used in the simulation. Some results of applying the traditional and improve NAS-RIF algorithm on these blurred images

\section{REFERENCES}

[1] Blind deconvolution subject to sparse representation for fluorescence microscopy Original Research Article Optics Communications, Volume 286, 1 January 2013, Pages 60-68 Yu Wang, Qionghai Dai, Qiang Cai, Peiyuan Guo, Zaiwen Liu

[2] PSF calibration patterns selection based on sensitivity analysis Original Research Article Microelectronic Engineering, Volume 112, December 2013, Pages282286 Thiago Figueiro, Mohamed Saib, Jean-Herve Tortai, Patrick Schiavone

[3] Total variation blind deconvolution employing split Bregman iteration Original Research Article Journal of Visual Communication and Image Representation, Volume 23, Issue 3, April 2012, Pages 409-417 Weihong Li, Quanli Li, Weiguo Gong, Shu Tang

[4] Blind restoration of real turbulence-degraded image with complicated backgrounds using anisotropic regularization Original Research Article Optics Communications, Volume 285, Issue 24, 1 November 2012, Pages 4977-4986 Hanyu Hong, Liangcheng Li, Tianxu Zhang

[5] Constrained iterations for blind deconvolution and convexity issues Original Research Article Journal of Computational and Applied Mathematics, Volume 197, Issue 1, 1 December 2006, Pages 29-43 Giulia Spaletta, Luca Caucci

\section{APPENDIX}

\section{Tables}

Table 1. The PSNR and MSE computed

\begin{tabular}{|l|c|c|c|}
\hline & TEXT & LENA & FLOWER \\
\hline MSE & 0.0688 & 0.4257 & 0.3241 \\
\hline PSNR & $59.7554 \mathrm{db}$ & $51.8401 \mathrm{db}$ & $49.6253 \mathrm{db}$ \\
\hline
\end{tabular}

\section{Figures}

Figure 1.Proposed blind image deconvolution method

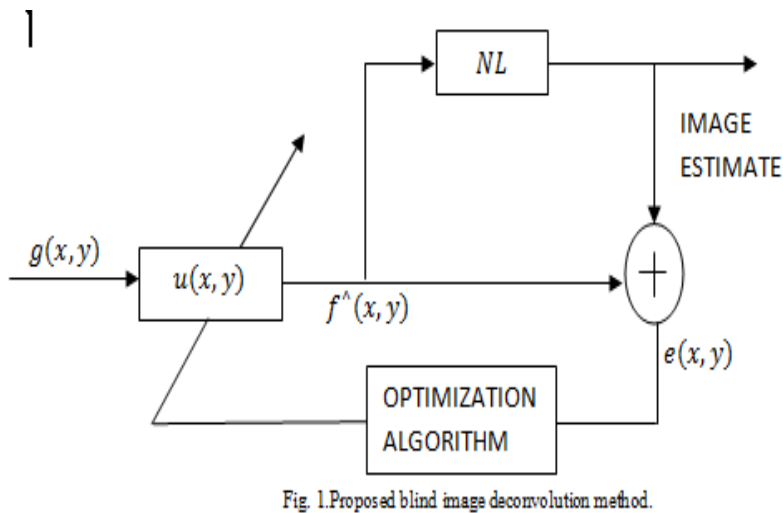

Figure 2.Example of a finite support image 
IMAGEFRAMEOF

UNDISTORED OBJECT

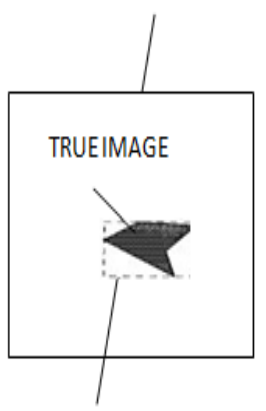

SUPPORTOF

TRUEOBJECT
IMAGEFRAMEOF

BLURRED OBJECT

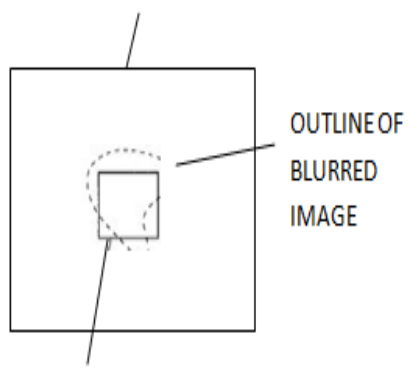

SUPPORTOF

TRUEOBJECT

Fig. 2.Example of a finite support mage.
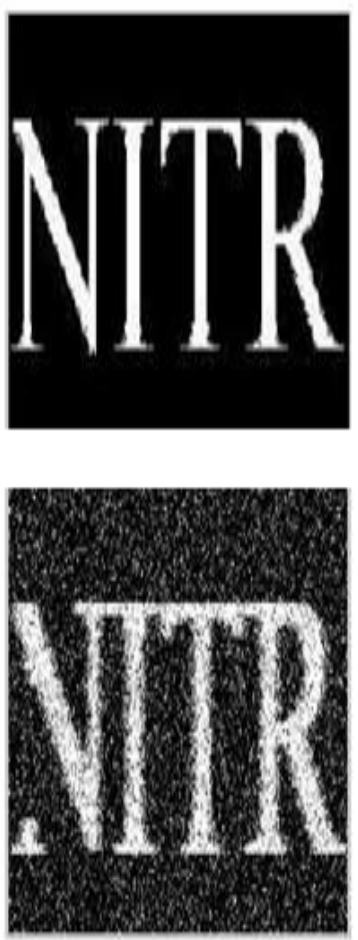
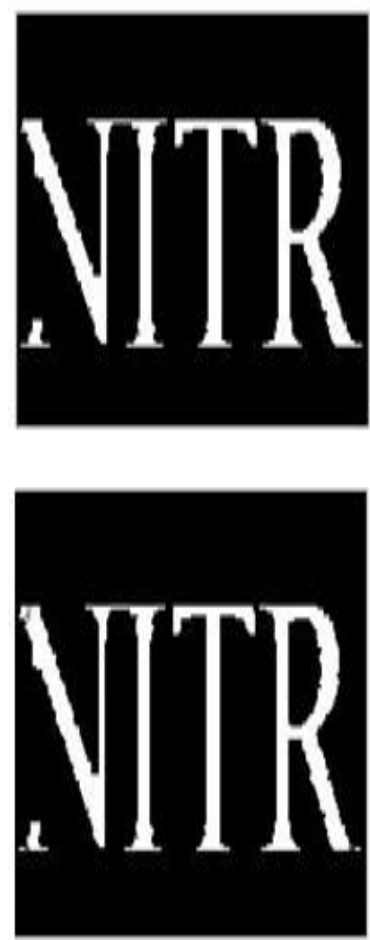

Fig. 3: (a) the Original Image. (b) Motion Blurred Image with angle $=0.2$ and Length $=7$ (c) The resulted image .
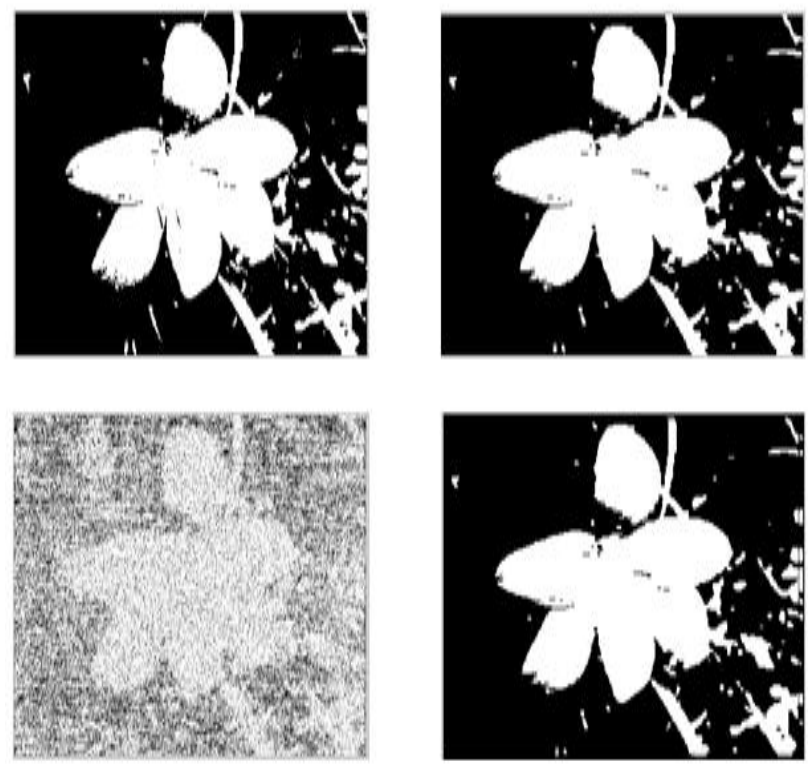

Fig. 4 :(g) The Original image in gray ,(i) The Blurre image with angle $=0.2$ and Length $=7 \quad$ (j) The Blurred image with noisy,(k) The resulted image.
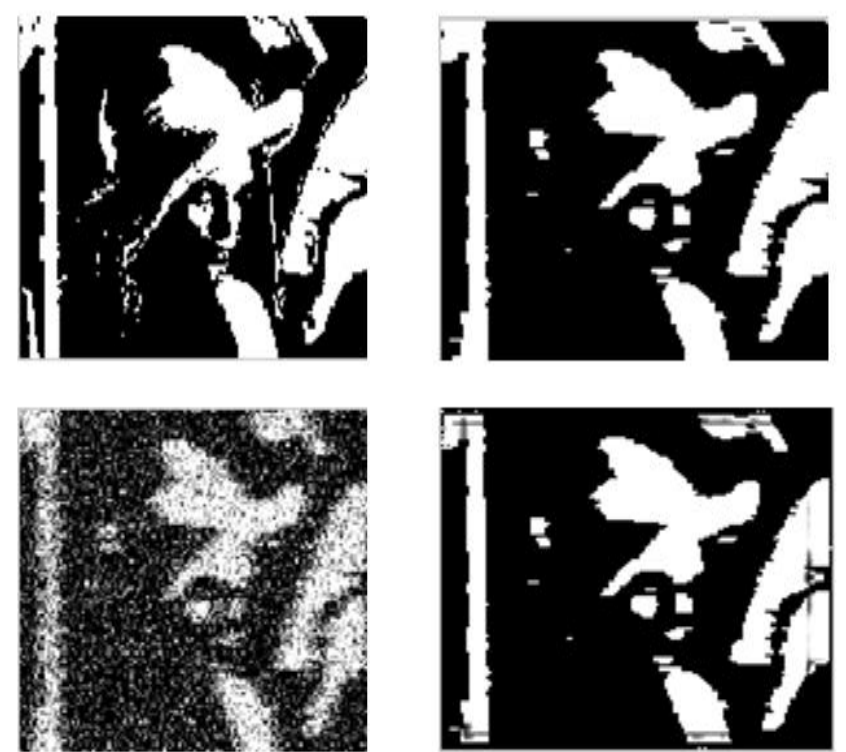

Fig. 5 : ( L) The Original image, (M) The Blurred image with angle $=0.2$ and Length $=7 \quad(O)$ The Blurred image with noisy,(P) The resulted image. 